\title{
A global phylogenetic analysis in order to determine the host species and geography dependent features present in the evolution of avian H9N2 Influenza Hemagglutinin
}

A complete phylogenetic analysis of all of the H9N2 hemagglutinin sequences that were collected between 1966 and 2012 was carried out in order to build a picture of the geographical and host specific evolution of the hemagglutinin protein. To improve the quality and applicability of the output data the sequences were divided into subsets based upon location and host species.

The phylogenetic analysis of hemagglutinin reveals that the protein has distinct lineages between China and the Middle East, and that wild birds in both regions retain a distinct form of the $\mathrm{H} 9$ molecule, from the same lineage as the ancestral hemagglutinin. The results add further evidence to the hypothesis that the current predominant H9N2 hemagglutinin lineage might have originated in Southern China. The study also shows that there are sampling problems that affect the reliability of this and any similar analysis. This raises questions about the surveillance of H9N2 and the need for wider sampling of the virus in the environment.

The results of this analysis are also consistent with a model where hemagglutinin has predominantly evolved by neutral drift punctuated by occasional selection events. These selective events have produced the current pattern of distinct lineages in the Middle East, Korea and China. This interpretation is in agreement with existing studies that have shown that there is widespread intra-country sequence evolution. 
1 Andrew R. Dalby

2 Faculty of Science and Technology, University of Westminster, 115 New Cavendish Street, Westminster, 3 W1W 6UW, UK.

4 Munir Iqbal

5 Avian Viral Diseases Programme, The Pirbright Institute, Compton Laboratory, Newbury, Berkshire. 6 RG20 7NN, UK

7 A.Dalby@westminster.ac.uk 


\section{PeerJ Reviewing Manuscript}

\section{Introduction}

9 The spread of avian influenza viruses (AIV) are a major cause of concern for global animal and

10 public health; these viruses are causing enormous economic losses as well as posing a credible

11 threat for pandemic emergence (Sorrell et al. 2009; Xu et al. 2004; Yu et al. 2011). There has

12 been an increase in the monitoring of disease outbreaks in wild and domestic birds, as well as in

13 other potential hosts such as swine and humans, but we still lack a coordinated global

14 surveillance network (Butler 2012). The Influenza A/H5N1 virus has been the main focus of

15 international monitoring after a series of recent outbreaks, but the emergence of the A/H1N1

16 pandemic virus "swine flu" in 2009 showed that other subtypes also pose a serious threat to

17 human health (Cao et al. 2009). Experiments have been carried out to determine the exact factors

18 of bird to human transmission and of droplet transmission of H9N2 viruses (Sorrell et al. 2009).

19 The H9N2 subtype is a variant of AIV usually associated with low pathogenicity. Due to the

20 lower pathogenicity phenotype of this virus, data collection has been very sporadic. There have

21 been outbreaks of H9N2 in flocks of domestic birds resulting in significant economic loss and

22 with high mortality rates of up to $60 \%$ reported during the epizootic of 1998-2001 in Iran (Nili \&

23 Asasi 2002). This subtype has also been shown to pass to pigs, ferrets and guinea pigs, as well as

24 to humans in a small number of cases (Butt et al. 2005; Cheng et al. 2011; Lin et al. 2000; Lv et

25 al. 2012; Peiris et al. 1999; Wan et al. 2008; Xu et al. 2004; Yu et al. 2008; Zhang et al. 2009).

26 Antibodies to the virus have also been found in a sero-epidemiological investigation of poultry

27 workers (Pawar et al. 2012; Wang et al. 2009). These cross species infections show that in the

28 future the virus may present a serious threat to human health.

29 The co-circulation of H9N2 with other H5N1, H7N3, H1N1 and H3N2 subtypes has resulted in

30 the emergence of novel reassortant viruses (Monne et al. 2013; Peiris et al. 2001; Sun et al. 2011).

31 The reassorted virus has been shown to possess increased virulence (Iqbal et al. 2009; Marshall et 
32 al. 2013). The recent emergence of a novel reassortant H7N9 virus containing internal genes from

33 the H9N2 virus is another of novel AIV in birds which has the capability of infecting humans,

34 with fatal consequences. However in the cases of reassortment human to human transmission has

35 not been demonstrated (Watanabe et al. 2013).

36 There have been a number of recent studies on the evolution of AIV that have incorporated

37 geographical data available from global influenza monitoring (Fusaro et al. 2011; Haase et al.

38 2010; LAM et al. 2012; Wallace et al. 2007). With the growth in the global monitoring efforts,

39 and the widespread use of cheaper DNA sequencing technology there has been a rapid expansion

40 in the number of available sequences. Previous phylogenetic studies of H9N2 hemagglutinin,

41 have focussed on sequences from a single location (Banks et al. 2000; Butt et al. 2010; Kim et al.

42 2006; Li et al. 2005; Song et al. 2011; Xu et al. 2007a). The largest previous phylogeographical

43 study was that of Fusaro et al. who surveyed all of the H9N2 viral segments from the Middle East

44 (Fusaro et al. 2011). Fusaro's study defined eight geographical regions covering the Middle East

45 and used maximum likelihood methods to construct the phylogenetic analysis. Bayesian methods

46 were used to evaluate the geographical clade distribution. This work has recently been extended

47 to take a more detailed look at viral evolution in Israel and to show that there have been

48 successive introductions from neighbouring countries (Davidson et al. 2014).

49 A large-scale phylogenetic analysis using eight viral gene segments from 571 complete genomic

50 sequences collected between 1966 and 2009 was carried out by Dong and co-workers (Dong et

51 al. 2011). Geographical details were not the focus of this study as the aim was to establish the

52 lineage structure and genotypes present in H9N2. That analysis revealed 74 lineages and 98

53 genotypes when re-assortment is taken into consideration, but they only identified 7 HA variants.

54 Investigations of the evolution of influenza A H3N2 hemagglutinin using evidence from flu

55 antigen evolution, have shown how the rates of evolution can vary between selection events 
56 (epochs) (Koelle et al. 2006; Smith et al. 2004; Thomas \& Hertz 2012; Wolf et al. 2006). Wagner

57 used this study to suggest a reconciliation between the selectionist and neutralist views in a

58 network based model (Wagner 2008). Wagner's model also shows that the order in which

59 mutations take place can have an effect on the selection of a group of mutations. In this way

60 random drift is punctuated by selective epochs. Using mathematical models, Bedford et al. have

61 shown that in $\mathrm{A} / \mathrm{H} 3 \mathrm{~N} 2$ sequence evolution is constrained by canalisation (Bedford et al. 2012).

62 This agrees with Wagner's hypothesis if there are times when there is limited drift but occasional

63 bursts where the organism escapes the canalisation (Thomas \& Hertz 2012; Wolf et al. 2006).

64 It is difficult to assess the performance of phylogenetic models. The methods are sensitive to the

65 distance measures used and this is reflected in the number of sites that can be compared and the

66 number of sequences in the study (Felsenstein \& Felenstein 2004). Clades should be

67 monophyletic if they are produced by divergent evolution (Page \& Holmes 2009). If a lineage is

68 geography and host specific then we would expect all of the sequences that form a clade to share

69 the same labels in terms of geography and the species in which they are found. Single base or

70 amino acid changes are ambiguous and could be a product of either divergence or convergence,

71 but larger conserved patterns, especially if they are non-consecutive in the sequence alignments

72 are good indicators of mutations that are responsible for differentiating between clades. These

73 patterns of change can then be examined in terms of their effects on protein structure and

74 function. Ultimately it is the biological function that determines how selection has been

75 responsible for clade differentiation.

76 The current study presents a comprehensive phylogenetic analysis of the H9N2 HA, that includes

77 sequences from all of the geographical regions where H9N2 has been reported. This investigation

78 shows how host species and geographical distribution have shaped the evolution of distinct

79 lineages of H9 HA. This reveals clades that are both geographically and host species dependent. 
80 From these analyses new hypotheses can be generated for more specific events such as

81 migrations or intra-species infections. 
82 Materials and Methods

83 The complete set of H9N2 hemagglutinin protein sequences were downloaded from the NCBI on

84 the $22^{\text {nd }}$ of May 2013. The search term used for searching the protein database was H9N2

85 hemagglutinin. The sequences were exported in FASTA format.

86 The complete set of H9N2 hemagglutinin nucleotide sequences were downloaded from the NCBI

87 on the $9^{\text {th }}$ of September 2013. The search term used was ((H9N2 hemagglutinin) NOT

88 precursor) NOT partial. The sequences were downloaded in FASTA format.

89 A dataset for the complete set of Korean H9N2 hemagglutinin sequences was downloaded from

90 the NCBI on the $15^{\text {th }}$ of June 2014. The search term used was ((H9N2 hemagglutinin) NOT H5)

91 Korea).

92 After removal of long truncations ( $>40$ amino-acid residues) as well as a group of sequences that

93 were actually from other AIV subtypes, the final protein dataset contained 2045 sequences, the

94 final nucleotide dataset contained 1052 sequences of which the Korean nucleotide subset

95 contained 64 sequences.

96 The edited protein and nucleotide datasets were then broken into sub-groups based on the

97 sequence annotations, using a short text matching program written in Perl. The data was split into

98 subsets based on geographical location and host species.

99 The geographical subsets were based on national boundaries except for China, which was divided

100 into its regions. Where a Chinese region had 10 sequences or less then phylogenetic analysis was

101 not carried out. National borders have been identified as barriers to influenza transmission and so

102 these are appropriate geographical subsets (Wallace \& Fitch 2008). A subset was created for the 
103 Americas including all of the North American and the single South American (Argentinian)

104 sequence.

105 Species trees were created for chicken, duck (including mallard), quail, pheasant and swine. In

106 the past it has not been common practice to produce species specific phylogenetic trees but a

107 recent paper by Worobey et al. also used this approach (Worobey et al. 2014). There are

108 numerous wild bird species and also some environmental samples, but these are often only

109 represented by single cases and so subsets were not created.

110 All of the sequence analysis and editing was carried out in MEGA 5.2 on a Windows 8 computer.

111 Both the protein and gene sequences were aligned using Muscle within the MEGA sequence

112 analysis package, using the default parameters (Edgar 2004; Tamura et al. 2011).

113 Model evaluation was carried out for the protein dataset within MEGA. This analysis showed that

114 the Jones-Taylor-Thornton model with gamma distributed rates amongst sites was the best model

115 (supplementary table 1) (Jones et al. 1992). Phylogenetic trees for the protein sequences were

116 then constructed using Maximum Likelihood within MEGA using the JTT + G substitution

117 model. Each model was tested with 500 bootstraps replicates. To make the calculations tractable

118 the heuristic nearest neighbour interchange was used. The initial tree was created with

119 neighbourhood joining.

120 Model evaluation for the corresponding nucleotide dataset showed that the Tamura-Nei

121 substitution model with gamma distributed rates amongst sites was the best performing model

122 (supplementary table 2) (Tamura \& Nei 1993). Phylogenetic trees for the gene sequences were

123 constructed using Maximum Likelihood within MEGA using the TN93 + G substitution model.

124 Models were tested with 500 bootstrap replicates. Smaller numbers of sequences in the gene 
125 dataset meant that it was possible to calculate the bootstrap values for the chicken trees, which 126 could not be calculated in the protein trees.

127 The amino acids responsible for clade formation were determined manually using the alignment 128 view within MEGA. This view shows only the amino acid changes relative to the conserved 129 sequence, and so it is a matter of scanning the sequences looking for substitutions that correspond 130 to the different clusters. 
131 All of the trees have been presented as cladograms rather than as phylograms because the study

132 uses a cladistic approach for the analysis. The topology (ordering of the clusters) is the most

133 significant factor in this investigation rather than the distances between groups. The removal of

134 distance data and also the bootstrap data from the figures improves the clarity of the diagrams,

135 but this omitted information is available in the supplementary files that include the full

136 phylogenetic analysis. Results and Discussion

\section{Global Nucleotide Phylogenetic Tree Analysis.}

138 A complete phylogenetic analysis of the nucleotide dataset was carried out. Computational limits

139 on memory and processor speed make it impossible to carry out a complete analysis on the

140 protein dataset (especially as it contains almost twice as many sequences). A condensed view of

141 the principal clades from the global nucleotide tree can be seen in figure 1. One noticeable

142 absence from the global nucleotide tree is the quail sequences. These were omitted during the

143 editing of the sequences to remove truncated sequences where the ends of the sequences were

144 missing. The virus can be broken into three main clusters labelled A, B and the Main Chinese

145 Clade. (The complete tree can be found in supplementary figure 1). There is also a small clade

146 (labelled C) that splits from the root of the tree between clades A and B. This clade contains

147 Chinese sequences that were isolated from chickens between 1999-2002. This topology agrees

148 with those from the existing literature except that there is some disagreement in the identification

149 of the lineages (Li et al. 2003; Peiris et al. 2001; Perk et al. 2006; Yu et al. 2008). Ji used a

150 lineage and sub-lineage nomenclature that identified four lineages and 2 sub-lineages

151 corresponding to Clade A (lineages 9.1, 9.2 and 9.3), Clade B (sub-lineage 9.4.1) and the main

152 Chinese Clade (sub-lineage 9.4.2). Huang and co-workers used a lineage naming system based on

153 the prototypes Y439, TY WS 66, G1 and Y280 (Huang et al. 2010). Y439, and TY WS 66

154 (Turkey, Wisconsin 1966) are both in clade A. G1 is in clade B and Y280 is in the main Chinese 
155 clade in China 1 1994-2010. The large study by Dong identified HK/G1/97, BJ/1/94, HK/289/78,

$156 \mathrm{HK} / \mathrm{AF} 157 / 92, \mathrm{KR} / 96323 / 96, \mathrm{DE} / 113 / 95$ and $\mathrm{WI} / 1 / 66$ as prototype sequences for the different

157 HA lineages (Dong et al. 2011). The G1 lineage and WI/1/66 lineages are well established and

158 correspond to Clade B and Clade A respectively. BJ/1/94 is in the same clade as Y280,

$159 \mathrm{HK} / 289 / 78, \mathrm{HK} / \mathrm{AF} 157 / 92$ and $\mathrm{DE} / 113 / 95$ are not in the current dataset because of truncations,

160 but there are sub-clades for Hong Kong duck sequences and Korean chicken sequences that

161 correspond to $\mathrm{HK} / 289 / 78$ and $\mathrm{KR} / 96323 / 96$ respectively. This suggests that it might be possible

162 to divide Clade a into further sub-clades but given the limited number of sequences sampled there

163 is insufficient evidence to be able to carry this out at present.

164 Within the Main Chinese clade there are a series of nested sub-clades labelled China 1-9 where

165 the annotations follow the correct date order, with the exception of clade 8 . In clade 8 there is a

166 single sequence from a chicken in the Shandong region collected in 1999, which is a probable

167 outlier. All of the other sequences within the clade are from 2003 onwards, which would be

168 consistent with the splitting dates for the other sub-clades. It is possible that this is an example of

169 convergent evolution between recent Chinese sequences and an earlier branching of the

170 phylogenetic tree, but a single sequence is insufficient evidence to corroborate this. An alternative

171 explanation is that this results from sequencing error but this is also unlikely given the large

172 number of bases that would have to be incorrectly identified (for an alignment between the

173 Shandong sequence, G1, G9/Y280, Wisconsin 1966 sequences see supplementary figure 2). It is

174 possible that this could be a database error, but again this seems unlikely given the provenance

175 and tracking systems within GenBank. In the absence of database error and a clear evolutionary

176 connection between this lone sequence and the rest of the clade does provide evidence for the

177 inadequate sampling of sequences. This nested structure of the Chinese sequences had previously

178 been reported by Song et al. (Song et al. 2011). 
179 The expanded tree (supplementary figure 1) shows that the geographical sampling has not been

180 systematic and that it has been carried out in a sporadic and haphazard manner. There are breaks

181 in the dates of sequence annotations in some of the clades that are homogeneous for location.

182 Date gaps in annotations at specific locations are likely to be a result of inadequate sampling

183 rather than reliable evidence for the loss and subsequent migratory return of the clade. The focus

184 on China because of outbreaks of H9N2 within flocks of domestic birds has resulted in a very

185 dense phylogenetic tree for the Chinese sequences, resulting in an artificially Chinese focused

186 distribution to the phylogenetic trees. There is only a single sequence from South America this is

187 the result of a sampling effect rather than reflecting the actual H9N2 distribution.

188 Clade A (figure 2) corresponds to wild bird infections that are distributed world-wide and include

189 examples from North America, South America, Europe and the Far East. This is an important

190 clade because it also contains the original sequence of the hemagglutinin from the H9N2 subtype

191 that was found in a turkey in Wisconsin in 1966. This clade also contains a number of recent

192 sequences from Korea the US and Europe and so this clade remains extant. The topology of this

193 clade disagrees with that from Kim and co-workers who constructed a tree for regions 1-1104

194 using DNA Star (Kim et al. 2006). The complete sequence for HA is over 1700 bases, and the

195 method used here is Maximum Likelihood, whereas DNA Star uses the less reliable UPGMA tree

196 generation algorithm within ClustalW. In their tree the recent Korean sequences were placed

197 outside clade A and beyond the Y280 sequences as a distinct clade. The bootstrap values are high

198 for this region of the tree (> 99\%) and it has geographical consistency with the rest of the clade,

199 and so the topology presented in the current paper is most likely to be correct.

200 Clade B (figure 3) contains mostly Middle Eastern sequences although there are a few Chinese

201 sequences that form a sub-clade. This clade corresponds to the extended G1 lineage used by

202 Fusaro et al. and Monne et al. (Monne et al. 2013) Iran and Israel are the two countries most 
203 strongly represented in this clade. There are also a number of sequences from the Arabian

204 peninsular and a large grouping from the Indian sub-continent.

205 The sub-lineage structure of Clade B shows that the sequences have evolved substantially from 206 the G1 prototype. The G1 prototype sub-clade became extinct in Iran in 2004 and in Israel in 207 2007. This was replaced by a new sub-lineage (labelled 725 sub-clade in figure 3) that appears to 208 have originated in chicken flocks in Iran in 1998, although it is only found in a large number of 209 Iranian samples after 2004. This sub-lineage is similar to that previously identified by Fusaro et 210 al. and Monne et al. (labelled cluster C in their papers) (Fusaro et al. 2011; Monne et al. 2013). 211 There is a sub-clade from the Indian subcontinent that originated in the Punjab/Haryana region in 212 2003. There is a linking clade than originated in Pakistan in 2004 before spreading back to Iran in 213 2009. The final sub-clade seems to have originated in the Arabian Peninsular in 2006 and 214 correspond to cluster B from the studies of Fusaro et al. and Monne et al. This sub-lineage then 215 spread to Israel and most recently to Egypt. This is in good agreement with the previous studies 216 on the origin of the Egyptian virus (Abdel-Moneim et al. 2012). The phylogenetic tree shows that 217 after the initial Egyptian outbreak in 2010 there has been a marked diversification in the 218 sequences.

219 Clade C (figure 4) is a Chinese clade that has annotated dates between 1999 and 2001. In the 220 phylogenetic analysis this clade falls between Clade A and Clade B. As there are only a small 221 number of sequences within the clade there is no discernable pattern to the distribution of the 222 sequences within the Chinese regions, although it appears to have originated in Guangdong in 223 1999. As there are no more sequences after those from 2002 this clade can be considered extinct.

224 The first Chinese clade contains the G9/Y280 lineage (figure 5). After the banning of live quail 225 from poultry markets the G1 lineage disappeared from Chinese poultry leaving only the G9/Y280 226 lineage (Choi et al. 2004). In the study of Li et al. four lineages were specified G1, TY/WS/66, 
227 Y439 and Beijing/1/94, but no sequences from G1 or Y439 were found in their sample (Li et al.

228 2005). Cong and co-workers identified two more lineages within this clade based on antigenic

229 studies and nucleotide phylogenetic trees (Cong et al. 2007). These are represented by prototype

230 sequences from Shanghai 1998 and a swine genotype. These lineages only cover a small number

231 of the possible sub-clades within this first Chinese Clade (figure 5). As no sequences have been

232 sampled from this clade since 2010 it is possible that the G7/Y280 lineage is now extinct, but this

233 hypothesis cannot be confirmed without a longer period of absence of viruses from this clade.

234 This study shows a series of new clades that had not been previously identified and that originate

235 in 1997 in Beijing. This is also the origin of the clade labelled China 2. All of the subsequent

236 nested Chinese clades are related to this Beijing sequence. Some sub-clades such as China 4,

237 China 5 and China 6 also appear to have become extinct and so there is good evidence for

238 successive selective sweeps through the viral population.

239 As discussed previously the individual unusual sequence from 1999 in China 8 sub-clade is

240 difficult to explain. It might suggest that there is considerable sequence diversity within these

241 nested clades, but because of the low number of individuals carrying a particular sequence it

242 might take a long-time for a sequence to become fixed sufficiently within the population to be

243 found by sporadic sampling. If this is true then all of the clades probably have a much earlier

244 origin and there is a long period before first detection. This causes some concern for tracking the

245 evolution of potential pandemic strains, as they might be circulating quite widely before they are

246 detected for the first time.

\section{Clade Analysis of the Geographical Subsets}

248 From the global phylogenetic tree three interesting geographical subsets were identified; the tree

249 for Korea because it is homogeneous to clade A, and the trees from Iran and Israel as they show 
250

251 features but these are difficult if not impossible to untangle and coherently explain because of the

252 limitations of sampling within the data, where there are only small numbers from some regions

253 and then large numbers from others.

254 Both the protein and nucleotide phylogenetic trees for Korea are shown in figure 6. There is a fair 255 agreement between the two trees but once again this emphasizes the problems of sampling and 256 the possible effects this can have on phylogenetic reconstruction. From the nucleotide tree it is 257 tempting to define a clade that contains only wild birds and that suddenly developed in 2005 , but 258 this clade is not present in the protein tree, and so it cannot be definitively assigned. There are 259 also some differences in the topology surrounding the swine flu case (marked with a red 260 diamond). Previous studies have investigated the effect of vaccination, which initially 261 suppressed the number of cases that were seen in 2008 before an increase in the number of cases 262 in 2009 (Park et al. 2011). From the trees presented here the period following the introduction of 263 vaccination does correspond to a period of diversification. All of the Korean sequences are from 264 clade A, which is the longest circulating lineage and previously it had not shown a wide diversity 265 of sequences. Vaccination is likely to have had an impact on hemagglutinin evolution, which can 266 be seen in the 2009-2010 clade (Lee \& Song 2013).

267 The protein and nucleotide phylogenetic trees for Israel and Iran are shown in Figure 7. In the 268 Iranian tree Clade 1 are duck sequences from Clade A. This is why this clade has the deepest 269 origin within the tree, but it does not contain the earliest sequences. This shows that wild birds 270 can introduce other lineages to a geographical region where another lineage currently dominates.

271 Clades 2 and 5 in the Iranian tree are the oldest clade from the G1-like lineage in these regions.

272 The G1 lineage appears to have originated in Hong Kong in 1997. The initial G1 clade (Clade 2)

273 becomes extinct in 2003 in the nucleotide tree and in 2005 in the protein tree indicating a 
274 selective sweep. This event coincides with the loss of sister Clades 1 and 2 in the Israeli protein

275 tree and Clade 1 in the nucleotide tree. These clades were assigned to cluster A by Fusaro et al.

276 and Monne et al. The second G1-like clade (clade 5, labelled sub-clade 725 in the global

277 phylogenetic tree, Fusaro/Monne Cluster D) continued to be found in Iran until 2009. This has no

278 equivalent in the Israeli trees, which seem to have inherited a G1-like lineage which originated in

279 the Indian sub-continent and then circulated in the Arabian peninsular (see the global

280 phylogenetic tree, supplementary figure1 and figure 3, Fusaro and Monne cluster B) before being

281 found in Israel in 2006/2007 (clade 3 in the nucleotide and protein trees) and in Iran in 2009

282 (clade 4 in the protein and nucleotide trees). A recent paper has shown that there have in fact been

283 several introductions of H9N2 to Israel from Jordan, and the Arabian Peninsula (Davidson et al.

284 2014). This newly introduced G1-like sub-lineage seems to have had a selective advantage over

285 the existing viral G1-like sub-lineages but this can only be confirmed by future sampling.

286 Subsequently this new sub-lineage has also spread to Egypt from Israel.

287 Clade Analysis of the Host Specific Subsets

288 The interesting host species subsets are those for ducks, quail and swine. Ducks are important as 289 a possible carrier of the virus between geographical regions and in acting as a reservoir species.

290 Quail have also been associated with acting as a host to allow re-assortment and viral evolution.

291 Finally swine are important because of the over-lapping glycosylation site specificity of swine

292 and human viruses, which suggests that they may act as an intermediate for transmission to

293 humans (Guo et al. 2005).

294 There are problems in interpreting the quail trees because of the absence of the Shantou 295 sequences from the nucleotide tree because of partial sequencing (figure 8). Shantou was the 296 main geographical location for the outbreak of H9N2 in quail between 2000 and 2005 (Xu et al. 297 2007a; Xu et al. 2007b). Live quail were banned from Chinese wet markets after a study had 
298 shown the link to the virus. In the literature it was found that by 2003 this had resulted in the G1-

299 like lineage disappearing from China but the data here show that it was still present in quail in the

300 Shantou region until 2005 (Choi et al. 2004). The trees show that quail are hosts for all of the

301 main lineages clade A, clade B (G1-like lineage) and the main Chinese clade. This would support

302 the theory that quail have been the host species responsible for the diversification of the H9N2

303 sub-type, especially given that the original sequence of G-like lineage that is the prototype

304 sequence for clade B was originally found in a Hong Kong quail. (Hossain et al. 2008). This also

305 fits with the epochal model of viral evolution proposed by Wagner (Wagner 2008). There are

306 periods of neutral drift, which are punctuated by selection events. Here the selection event is the

307 formation of a new lineage within a different host species after a long period of neutral drift

308 within clade A.

309 Like quail ducks provide a host for the clade A and main Chinese clade viral lineages (figure 9).

310 There is only a single example of a clade B sequence in ducks, and so they might not be very

311 effective carriers of this virus or this might be explained by their geographical exposure to that

312 lineage (Perez et al. 2003). Ducks are of concern as a host species as they can contribute to the

313 spread of this lineage over a wider geographical region. In the global phylogenetic tree sequences

314 from ducks are often found clustered with those from quail showing that there is frequent transfer

315 between the two hosts.

316 Most of the swine cases have been within the main Chinese clade of sequences (figure 10). There

317 is a single sequence from Korea in 2004 that belongs to clade A. This is important in guiding how

318 we monitor disease outbreaks because it shows that the most geographically widely distributed

319 clade can also produce infection in pigs. The swine flu epidemic of H1N1 originated in Mexico

320 whereas China had been the main focus of surveillance, because of the frequency and previous

321 circulation of the virus. This is also true of H9N2 monitoring which is focused on Southeast 


\section{PeerJ Reviewing Manuscript}

322 Asia, but shows that more widespread monitoring is important in the early detection of

323 pandemics.

324 There is no obvious geographical or temporal pattern in infection in pigs and there are multiple

325 transmissions between birds and pigs that produce a tree with many different sub-clades usually

326 with only a small number of members. This is consistent with their not being a widespread

327 circulation of the virus within pigs, which would give a larger homogeneous cluster of swine

328 viruses from different times and locations due to pig to pig transmission. Previously five amino

329 acids changes had been identified as being swine specific, but none of these were conserved

330 within the swine sequences or even within a single swine clade (Xu et al. 2004). There are a few

331 cases of the S145N mutation that has been identified to change the antibody epitope but this

332 again is sporadic (Ping et al. 2008).

\section{Identifying the Amino Acid Changes Responsible for Clade Formation}

334 The x-ray crystal structures are available for the H9 hemagglutinin and so it is possible to map

335 the amino acid changes responsible for differentiating between the different clades onto the

336 structure (Ha et al. 2001; Ha et al. 2002). The region between amino acids 128 and 275 makes up

337 the receptor subdomain. This domain is responsible for binding to the cellular membrane as part

338 of viral invasion. The stem domain is made up of the first 60 amino acids of the $\mathrm{N}$-terminus and

339 the final 275 amino acids in the C-terminus of which the last 221 are cleaved by proteolysis of

340 the precursor protein at a conserved arginine to produce a second peptide chain. In between these

341 domains is the remains of a catalytic domain - the vestigial enzyme domain (Ha et al. 2002). The

342 amino acids that are specific to the four most distinct clades are given in table 1 . 
343 The presence of a four key amino acids has been shown to be essential for droplet transmission of

344 the virus H183, A189, E190 and L226 that correspond to residues H191, A197, E198, and L234

345 in the H9 numbering (Sorrell et al. 2009).

346 There are 17 amino acids that distinguish between Clade A and the consensus sequence, only

347 three of these are in the receptor domain and so the majority are in the stem domains. Three

348 mutations are in the enzymatic domain of which the most significant of which is the replacement

349 of a serine at residue 127 with an asparagine as this is on the boundary of the domain and this

350 creates another potential glycosylation site. The receptor domain changes are Q164H, R180E

351 and T206A. Of these the replacement of the basic arginine group by an acidic glutamic acid is the

352 most interesting, because of the change in polarity, none of the amino acid changes affected either

353 the glycosylation sites or altered the residues that were identified as key to viral droplet

354 transmission. Only T8A had been previously identified as an important change when it was

355 shown to be involved in host specificity (Perez et al. 2003)

356 There are 26 amino acid changes that distinguish Clade B. Eleven of these changes can be found

357 in the receptor subdomain. Many of the changes are between leucine, isoleucine and valine.

358 These are conservative changes that preserve the hydrophobicity of the amino acid but change the

359 steric interactions. Another significant proportion of the changes is substitutions to threonine

360 from serine or valine. Three of the serine to threonine changes occur in the receptor domain and

361 this might reflect an altered binding affinity for a larger binding partner. Position A168 had been

362 identified previously as under positive selection (Fusaro et al. 2011). This is supported by the

363 mutation to leucine in this clade. Of the key amino acid changes required for droplet infection

364 only the N191H mutation is clade specific.

365 The amino acid changes responsible for differentiating between clades gives some support to

366 previous studies that have tried to identify residues that are under selection (Fusaro et al. 2011). 
367 However most of the clade specific changes have not been previously identified in the literature 368 on the evolution of antigenicity (Kaverin et al. 2004; Skehel \& Wiley 2000). The amino acids 369 responsible for glycosylation are conserved throughout the phylogenetic trees, although some of

370 the amino acid changes introduce new asparagine residues, which could be new sites for

371 glycosylation (Guo et al. 2000; Zhang et al. 2004). There are a very large number of sequences

372 with the L234 substitution required for droplet infection but these are not specific to any of the 373 major clades and this shows that the mutation has arisen multiple times. 


\section{Conclusions}

375 The sequence databases are growing at a hyperbolic rate, and with next generation sequencing

376 this level of growth is likely to continue for the foreseeable future. There are two challenges for

377 dealing with this data. The first is computational that requires improved algorithms and

378 implementations especially as we are moving to more computationally intensive methods of

379 analysis. The second is the quality of the data collection itself. Currently data collection is not

380 systematic and this seriously affects the reliability of the models that can be built. Sampling is

381 badly skewed to certain geographical locations, China being a prime example, while others are

382 ignored (Africa and South America). Surveillance of wild birds is particularly problematic.

383 Where there have been international efforts such as in the European Union study of the spread of

384 H5N1 in wild birds, even this was incomplete with no data from Spain and Eastern Europe, and

385 only partial data for France and Germany (Hesterberg et al. 2009).

386 There are also problems with partial and truncated sequences, as these often have to be excluded

387 from analysis. This is becoming less of a problem as sequencing methods become cheaper and so 388 complete sequences become more widely available.

389 Another area where there needs to be a significant improvement is in the quality of the sequence

390 annotations in the databases. For effective phylogeographic analysis it is important that future

391 data should be annotated with as much geographic data as is possible, this must include GPS

392 coordinates and further GIS (geographic information system) information to include habitat and

393 urbanisation measurements would be ideal (Scotch et al. 2011; Yasué et al. 2006).

394 In this paper there are clearly three different principal clades; Clade A - Wisconsin like, Clade B 395 - G1-like and the Main Chinese Cluster - Y280-like, but it is not clear when a new lineage has

396 arisen and when they are no longer "like" the prototype sequences. At the sub-lineage level the 
397 assignment of cluster and clades strongly depends on the sampling of the sequences and this has

398 produced some conflicts between different assignments in the literature. Geography is a much

399 stronger determinant of lineage rather than avian host, which seems to provide a much weaker

400 barrier to spread of the virus. However there is a definite barrier between bird species and pigs as

401 hosts.

402 The clade analysis has provided insight into the functional and structural evolution of the protein.

403 There is only a limited overlap between the residues identified in this study as important in clade

404 differentiation and those identified as significant in the existing literature. There is therefore a

405 need for further investigation of the functionality of these newly identified amino acid changes.

\section{Acknowledgments}

407 AD would like to thank Dr Lorna Tinworth for her careful reading of the manuscript, the four

408 anonymous referees for their helpful comments on the current submission and Prof. Stronzo

409 Bestiale for his comments on an earlier draft of the manuscript. 
411

412

413

414

415

416

417

418

419

420

421

422

423

424

425

426

427

428

429

430

431

432

433

434

435

436

437

438

439

440

441

442

443

444

445

446

447

448

449

450

451

452

453

454

455

456

457

458
Abdel-Moneim AS, Afifi MA, and El-Kady MF. 2012. Isolation and mutation trend analysis of influenza A virus subtype H9N2 in Egypt. Virol J 9:173.

Banks J, Speidel E, Harris P, and Alexander D. 2000. Phylogenetic analysis of influenza A viruses of H9 haemagglutinin subtype. Avian pathology 29:353359.

Bedford T, Rambaut A, and Pascual M. 2012. Canalization of the evolutionary trajectory of the human influenza virus. BMC biology 10:38.

Butler D. 2012. Flu surveillance lacking. Nature 483:520-522.

Butt AM, Siddique S, Idrees M, and Tong Y. 2010. Avian influenza A (H9N2): computational molecular analysis and phylogenetic characterization of viral surface proteins isolated between 1997 and 2009 from the human population. Virol J 7:319.

Butt KM, Smith GJ, Chen H, Zhang LJ, Leung YH, Xu KM, Lim W, Webster RG, Yuen KY, Peiris JS, and Guan Y. 2005. Human infection with an avian H9N2 influenza A virus in Hong Kong in 2003. J Clin Microbiol 43:5760-5767.

Cao B, Li X-W, Mao Y, Wang J, Lu H-Z, Chen Y-S, Liang Z-A, Liang L, Zhang S-J, and Zhang B. 2009. Clinical features of the initial cases of 2009 pandemic influenza A (H1N1) virus infection in China. New England Journal of Medicine 361:2507-2517.

Cheng VC, Chan JF, Wen X, Wu W, Que T, Chen H, Chan K, and Yuen K. 2011. Infection of immunocompromised patients by avian H9N2 influenza A virus. Journal of Infection 62:394-399.

Choi Y, Ozaki H, Webby R, Webster R, Peiris J, Poon L, Butt C, Leung Y, and Guan Y. 2004. Continuing evolution of H9N2 influenza viruses in Southeastern China.J Virol 78:8609-8614.

Cong YL, Pu J, Liu QF, Wang S, Zhang GZ, Zhang XL, Fan WX, Brown EG, and Liu JH. 2007. Antigenic and genetic characterization of H9N2 swine influenza viruses in China. J Gen Virol 88:2035-2041.

Davidson I, Fusaro A, Heidari A, Monne I, and Cattoli G. 2014. Molecular evolution of H9N2 avian influenza viruses in Israel. Virus genes 48:457-463.

Dong G, Luo J, Zhang H, Wang C, Duan M, Deliberto TJ, Nolte DL, Ji G, and He H. 2011. Phylogenetic diversity and genotypical complexity of H9N2 influenza A viruses revealed by genomic sequence analysis. PloS one 6:e17212.

Edgar RC. 2004. MUSCLE: multiple sequence alignment with high accuracy and high throughput. Nucleic acids research 32:1792-1797.

Felsenstein J, and Felenstein J. 2004. Inferring phylogenies: Sinauer Associates Sunderland.

Fusaro A, Monne I, Salviato A, Valastro V, Schivo A, Amarin NM, Gonzalez C, Ismail MM, Al-Ankari A-R, and Al-Blowi MH. 2011. Phylogeography and evolutionary history of reassortant H9N2 viruses with potential human health implications. J Virol 85:8413-8421.

Guo YJ, Krauss S, Senne DA, Mo IP, Lo KS, Xiong XP, Norwood M, Shortridge KF, Webster RG, and Guan Y. 2000. Characterization of the pathogenicity of members of the newly established H9N2 influenza virus lineages in Asia. Virology 267:279-288.

Guo YJ, Wen LY, Zhang Y, Wan M, Guo JF, Li Z, and Shu YL. 2005. [Do pigs play a role in human infection with avian influenza A H9N2 viruses]. Zhonghua Shi Yan He Lin Chuang Bing Du Xue Za Zhi 19:106-109. 
459

460

461

462

463

464

465

466

467

468

469

470

471

472

473

474

475

476

477

478

479

480

481

482

483

484

485

486

487

488

489

490

491

492

493

494

495

496

497

498

499

500

501

502

503

504

505

506

507

508

509

510

Ha Y, Stevens DJ, Skehel JJ, and Wiley DC. 2001. X-ray structures of H5 avian and H9 swine influenza virus hemagglutinins bound to avian and human receptor analogs. Proceedings of the National Academy of Sciences 98:11181-11186.

Ha Y, Stevens DJ, Skehel JJ, and Wiley DC. 2002. H5 avian and H9 swine influenza virus haemagglutinin structures: possible origin of influenza subtypes. The EMBO journal 21:865-875.

Haase M, Starick E, Fereidouni S, Strebelow G, Grund C, Seeland A, Scheuner C, Cieslik D, Smietanka K, and Minta Z. 2010. Possible sources and spreading routes of highly pathogenic avian influenza virus subtype H5N1 infections in poultry and wild birds in Central Europe in 2007 inferred through likelihood analyses. Infection, Genetics and Evolution 10:1075-1084.

Hesterberg U, Harris K, Stroud D, Guberti V, Busani L, Pittman M, Piazza V, Cook A, and Brown I. 2009. Avian influenza surveillance in wild birds in the European Union in 2006. Influenza Other Respir Viruses 3:1-14.

Hossain MJ, Hickman D, and Perez DR. 2008. Evidence of expanded host range and mammalian-associated genetic changes in a duck H9N2 influenza virus following adaptation in quail and chickens. PLoS One 3:e3170.

Huang Y, Hu B, Wen X, Cao S, Gavrilov BK, Du Q, Khan MI, and Zhang X. 2010. Diversified reassortant H9N2 avian influenza viruses in chicken flocks in northern and eastern China. Virus Res 151:26-32.

Iqbal M, Yaqub T, Reddy K, and McCauley JW. 2009. Novel genotypes of H9N2 influenza A viruses isolated from poultry in Pakistan containing NS genes similar to highly pathogenic H7N3 and H5N1 viruses. PLoS One 4:e5788.

Jones DT, Taylor WR, and Thornton JM. 1992. The rapid generation of mutation data matrices from protein sequences. Computer applications in the biosciences: CABIOS 8:275-282.

Kaverin NV, Rudneva IA, llyushina NA, Lipatov AS, Krauss S, and Webster RG. 2004. Structural differences among hemagglutinins of influenza A virus subtypes are reflected in their antigenic architecture: analysis of H9 escape mutants. J Virol 78:240-249.

Kim JA, Cho SH, Kim HS, and Seo SH. 2006. H9N2 influenza viruses isolated from poultry in Korean live bird markets continuously evolve and cause the severe clinical signs in layers. Vet Microbiol 118:169-176.

Koelle K, Cobey S, Grenfell B, and Pascual M. 2006. Epochal evolution shapes the phylodynamics of interpandemic influenza A (H3N2) in humans. Science 314:1898-1903.

LAM TTY, HON CC, Lemey P, Pybus OG, Shi M, Tun HM, Li J, Jiang J, Holmes EC, and LEUNG FCC. 2012. Phylodynamics of H5N1 avian influenza virus in Indonesia. Molecular ecology 21:3062-3077.

Lee DH, and Song CS. 2013. H9N2 avian influenza virus in Korea: evolution and vaccination. Clin Exp Vaccine Res 2:26-33.

Li C, Yu K, Tian G, Yu D, Liu L, Jing B, Ping J, and Chen H. 2005. Evolution of H9N2 influenza viruses from domestic poultry in Mainland China. Virology 340:7083.

Li KS, Xu KM, Peiris JS, Poon LL, Yu KZ, Yuen KY, Shortridge KF, Webster RG, and Guan Y. 2003. Characterization of H9 subtype influenza viruses from the ducks of southern China: a candidate for the next influenza pandemic in humans? J Virol 77:6988-6994.

Lin YP, Shaw M, Gregory V, Cameron K, Lim W, Klimov A, Subbarao K, Guan Y, Krauss S, Shortridge K, Webster R, Cox N, and Hay A. 2000. Avian-to-human transmission of H9N2 subtype influenza A viruses: relationship between H9N2 and H5N1 human isolates. Proc Natl Acad Sci U S A 97:9654-9658. 
511

512

513

514

515

516

517

518

519

520

521

522

523

524

525

526

527

528

529

530

531

532

533

534

535

536

537

538

539

540

541

542

543

544

545

546

547

548

549

550

551

552

553

554

555

556

557

558

559

560

561

562

563
Lv J, Wei B, Yang Y, Yao M, Cai Y, Gao Y, Xia X, Zhao X, Liu Z, Li X, Wang H, Yang H, Roesler U, Miao Z, and Chai T. 2012. Experimental transmission in guinea pigs of H9N2 avian influenza viruses from indoor air of chicken houses. Virus Res 170:102-108.

Marshall N, Priyamvada L, Ende Z, Steel J, and Lowen AC. 2013. Influenza virus reassortment occurs with high frequency in the absence of segment mismatch. PLoS pathogens 9:e1003421.

Monne I, Hussein HA, Fusaro A, Valastro V, Hamoud MM, Khalefa RA, Dardir SN, Radwan MI, Capua I, and Cattoli G. 2013. H9N2 influenza A virus circulates in H5N1 endemically infected poultry population in Egypt. Influenza Other Respir Viruses 7:240-243.

Nili H, and Asasi K. 2002. Natural cases and an experimental study of H9N2 avian influenza in commercial broiler chickens of Iran. Avian Pathol 31:247-252.

Page RD, and Holmes EC. 2009. Molecular evolution: a phylogenetic approach: John Wiley \& Sons.

Park KJ, Kwon H-i, Song M-S, Pascua PNQ, Baek YH, Lee JH, Jang H-L, Lim J-Y, Mo I-P, and Moon H-J. 2011. Rapid evolution of low-pathogenic H9N2 avian influenza viruses following poultry vaccination programmes. Journal of General Virology 92:36-50.

Pawar SD, Tandale BV, Raut CG, Parkhi SS, Barde TD, Gurav YK, Kode SS, and Mishra AC. 2012. Avian influenza H9N2 seroprevalence among poultry workers in Pune, India, 2010. PLoS One 7:e36374.

Peiris JS, Guan Y, Markwell D, Ghose P, Webster RG, and Shortridge KF. 2001. Cocirculation of avian H9N2 and contemporary "human" H3N2 influenza A viruses in pigs in southeastern China: potential for genetic reassortment? J Virol 75:9679-9686.

Peiris M, Yuen KY, Leung CW, Chan KH, Ip PL, Lai RW, Orr WK, and Shortridge KF. 1999. Human infection with influenza H9N2. Lancet 354:916-917.

Perez D, Webby R, Hoffmann E, and Webster R. 2003. Land-based birds as potential disseminators of avian/mammalian reassortant influenza A viruses. Avian Dis 47:1114-1117.

Perk S, Banet-Noach C, Shihmanter E, Pokamunski S, Pirak M, Lipkind M, and Panshin A. 2006. Genetic characterization of the H9N2 influenza viruses circulated in the poultry population in Israel. Comp Immunol Microbiol Infect Dis 29:207223.

Ping J, Li C, Deng G, Jiang Y, Tian G, Zhang S, Bu Z, and Chen H. 2008. Single-aminoacid mutation in the HA alters the recognition of H9N2 influenza virus by a monoclonal antibody. Biochem Biophys Res Commun 371:168-171.

Scotch M, Sarkar IN, Mei C, Leaman R, Cheung K-H, Ortiz P, Singraur A, and Gonzalez G. 2011. Enhancing phylogeography by improving geographical information from GenBank. Journal of biomedical informatics 44:S44-S47.

Skehel J, and Wiley DC. 2000. Receptor binding and membrane fusion in virus entry: the influenza hemagglutinin. Annual review of biochemistry 69:531-569.

Smith DJ, Lapedes AS, de Jong JC, Bestebroer TM, Rimmelzwaan GF, Osterhaus AD, and Fouchier RA. 2004. Mapping the antigenic and genetic evolution of influenza virus. Science 305:371-376.

Song Xf, Han P, and Chen YPP. 2011. Genetic variation of the hemagglutinin of avian influenza virus H9N2. J Med Virol 83:838-846.

Sorrell EM, Wan H, Araya Y, Song H, and Perez DR. 2009. Minimal molecular constraints for respiratory droplet transmission of an avian-human H9N2 influenza A virus. Proc Natl Acad Sci U S A 106:7565-7570.

Sun Y, Qin K, Wang J, Pu J, Tang Q, Hu Y, Bi Y, Zhao X, Yang H, and Shu Y. 2011. High genetic compatibility and increased pathogenicity of reassortants derived 
564

565

566

567

568

569

570

571

572

573

574

575

576

577

578

579

580

581

582

583

584

585

586

587

588

589

590

591

592

593

594

595

596

597

598

599

600

601

602

603

604

605

606

607

608

609

610

611

612

613

614

615 from avian H9N2 and pandemic H1N1/2009 influenza viruses. Proceedings of the National Academy of Sciences 108:4164-4169.

Tamura K, and Nei M. 1993. Estimation of the number of nucleotide substitutions in the control region of mitochondrial DNA in humans and chimpanzees. Mol Biol Evol 10:512-526.

Tamura K, Peterson D, Peterson N, Stecher G, Nei M, and Kumar S. 2011. MEGA5: molecular evolutionary genetics analysis using maximum likelihood, evolutionary distance, and maximum parsimony methods. Mol Biol Evol 28:2731-2739.

Thomas PG, and Hertz T. 2012. Constrained evolution drives limited influenza diversity. BMC biology 10:43.

Wagner A. 2008. Neutralism and selectionism: a network-based reconciliation. Nature Reviews Genetics 9:965-974.

Wallace RG, and Fitch WM. 2008. Influenza A H5N1 immigration is filtered out at some international borders. PLoS One 3:e1697.

Wallace RG, HoDac H, Lathrop RH, and Fitch WM. 2007. A statistical phylogeography of influenza A H5N1. Proceedings of the National Academy of Sciences 104:4473-4478.

Wan H, Sorrell EM, Song H, Hossain MJ, Ramirez-Nieto G, Monne I, Stevens J, Cattoli G, Capua I, Chen LM, Donis RO, Busch J, Paulson JC, Brockwell C, Webby R, Blanco J, Al-Natour MQ, and Perez DR. 2008. Replication and transmission of H9N2 influenza viruses in ferrets: evaluation of pandemic potential. PLoS One 3:e2923.

Wang M, Fu CX, and Zheng BJ. 2009. Antibodies against $\mathrm{H} 5$ and $\mathrm{H} 9$ avian influenza among poultry workers in China. N Engl J Med 360:2583-2584.

Watanabe T, Kiso M, Fukuyama S, Nakajima N, Imai M, Yamada S, Murakami S, Yamayoshi S, Iwatsuki-Horimoto K, and Sakoda Y. 2013. Characterization of H7N9 influenza A viruses isolated from humans. Nature 501:551-555.

Wolf YI, Viboud C, Holmes EC, Koonin EV, and Lipman DJ. 2006. Long intervals of stasis punctuated by bursts of positive selection in the seasonal evolution of influenza A virus. Biol Direct 1:357-360.

Worobey M, Han G-Z, and Rambaut A. 2014. A synchronized global sweep of the internal genes of modern avian influenza virus. Nature.

Xu C, Fan W, Wei R, and Zhao H. 2004. Isolation and identification of swine influenza recombinant A/Swine/Shandong/1/2003(H9N2) virus. Microbes Infect 6:919925.

Xu KM, Li KS, Smith GJ, Li JW, Tai H, Zhang JX, Webster RG, Peiris JS, Chen H, and Guan Y. 2007a. Evolution and molecular epidemiology of H9N2 influenza A viruses from quail in southern China, 2000 to 2005. J Virol 81:2635-2645.

Xu KM, Smith GJ, Bahl J, Duan L, Tai H, Vijaykrishna D, Wang J, Zhang JX, Li KS, Fan XH, Webster RG, Chen H, Peiris JS, and Guan Y. 2007b. The genesis and evolution of H9N2 influenza viruses in poultry from southern China, 2000 to 2005. J Virol 81:10389-10401.

Yasué M, Feare CJ, Bennun L, and Fiedler W. 2006. The epidemiology of H5N1 avian influenza in wild birds: why we need better ecological data. BioScience 56:923-929.

Yu H, Hua RH, Wei TC, Zhou YJ, Tian ZJ, Li GX, Liu TQ, and Tong GZ. 2008. Isolation and genetic characterization of avian origin H9N2 influenza viruses from pigs in China. Vet Microbiol 131:82-92.

Yu H, Zhou Y-J, Li G-X, Ma J-H, Yan L-P, Wang B, Yang F-R, Huang M, and Tong G-Z. 2011. Genetic diversity of H9N2 influenza viruses from pigs in China: a potential threat to human health? Vet Microbiol 149:254-261. 
616

617

618

619

620

621

622

623
Zhang M, Gaschen B, Blay W, Foley B, Haigwood N, Kuiken C, and Korber B. 2004. Tracking global patterns of $\mathrm{N}$-linked glycosylation site variation in highly variable viral glycoproteins: HIV, SIV, and HCV envelopes and influenza hemagglutinin. Glycobiology 14:1229-1246.

Zhang P, Tang Y, Liu X, Liu W, Zhang X, Liu H, Peng D, Gao S, Wu Y, Zhang L, Lu S, and Liu X. 2009. A novel genotype H9N2 influenza virus possessing human H5N1 internal genomes has been circulating in poultry in eastern China since 1998. J Virol 83:8428-8438. 
Figure 1

Phylogenetic Overview

A compressed view of the complete phylogenetic tree with the major clades shown in a condensed format.

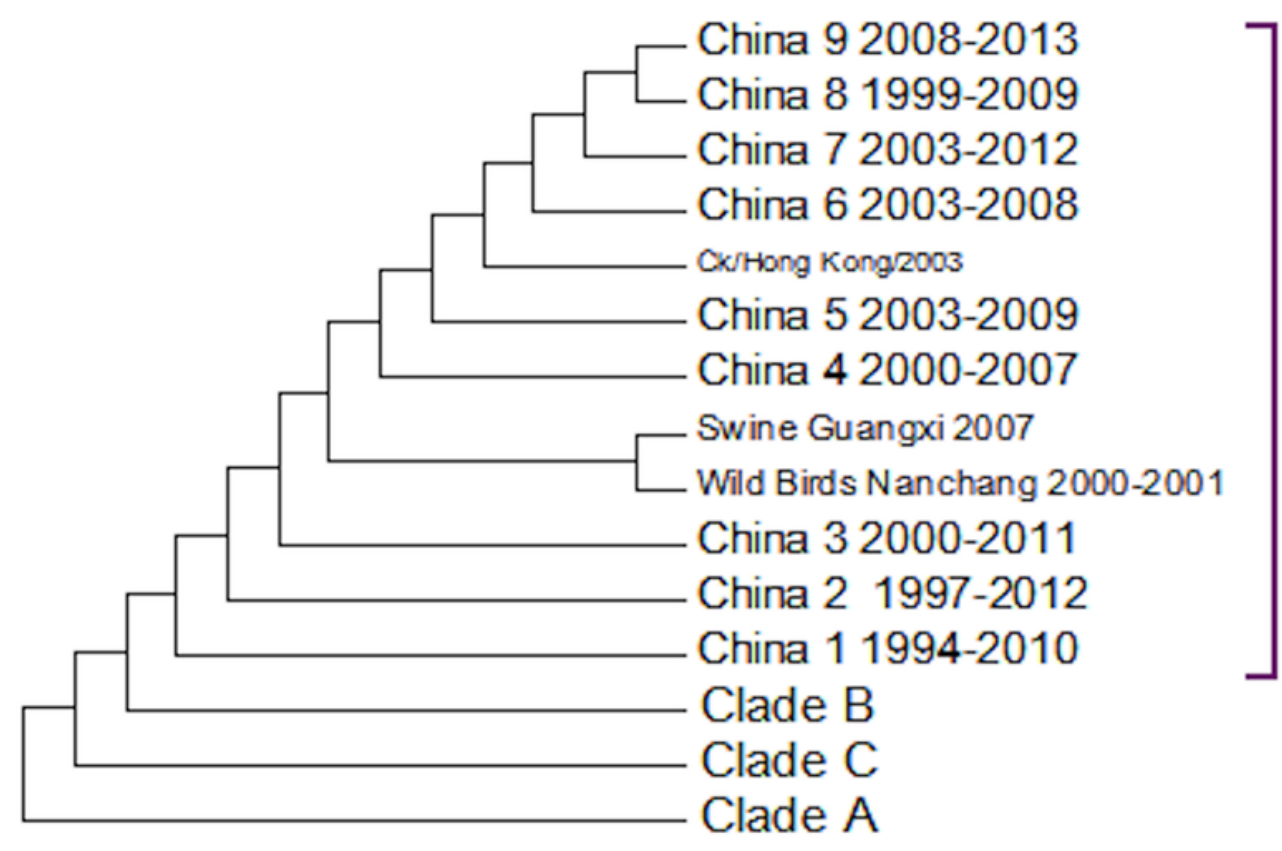

Main Chinese Clade 


\section{Figure 2}

\section{Clade A}

\section{This is the original lineage of H9N2 that was first isolated in a turkey in Wisconsin in 1966.}

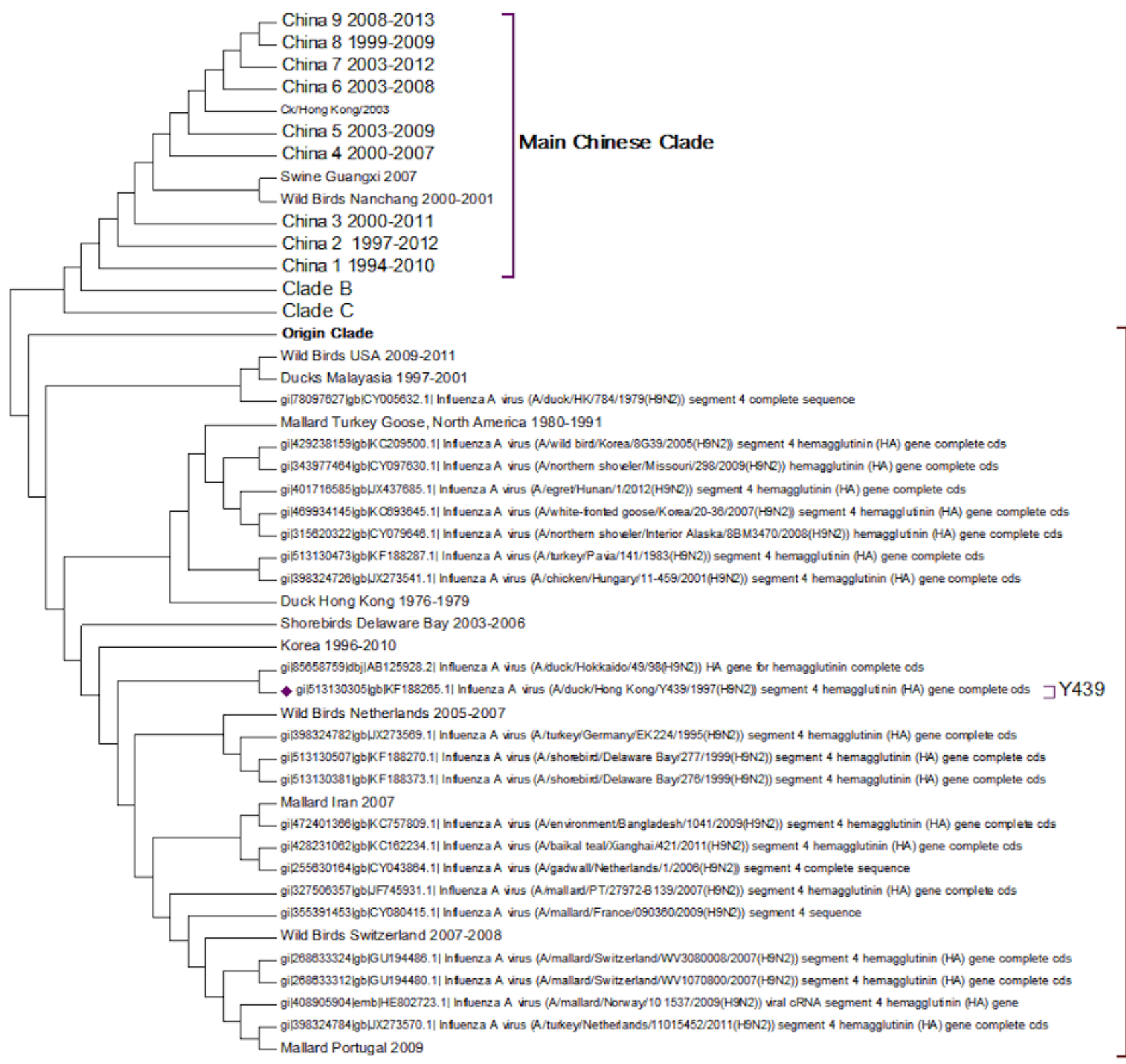


Figure 3

\section{Clade B}

This is also known as the G1 lineage.

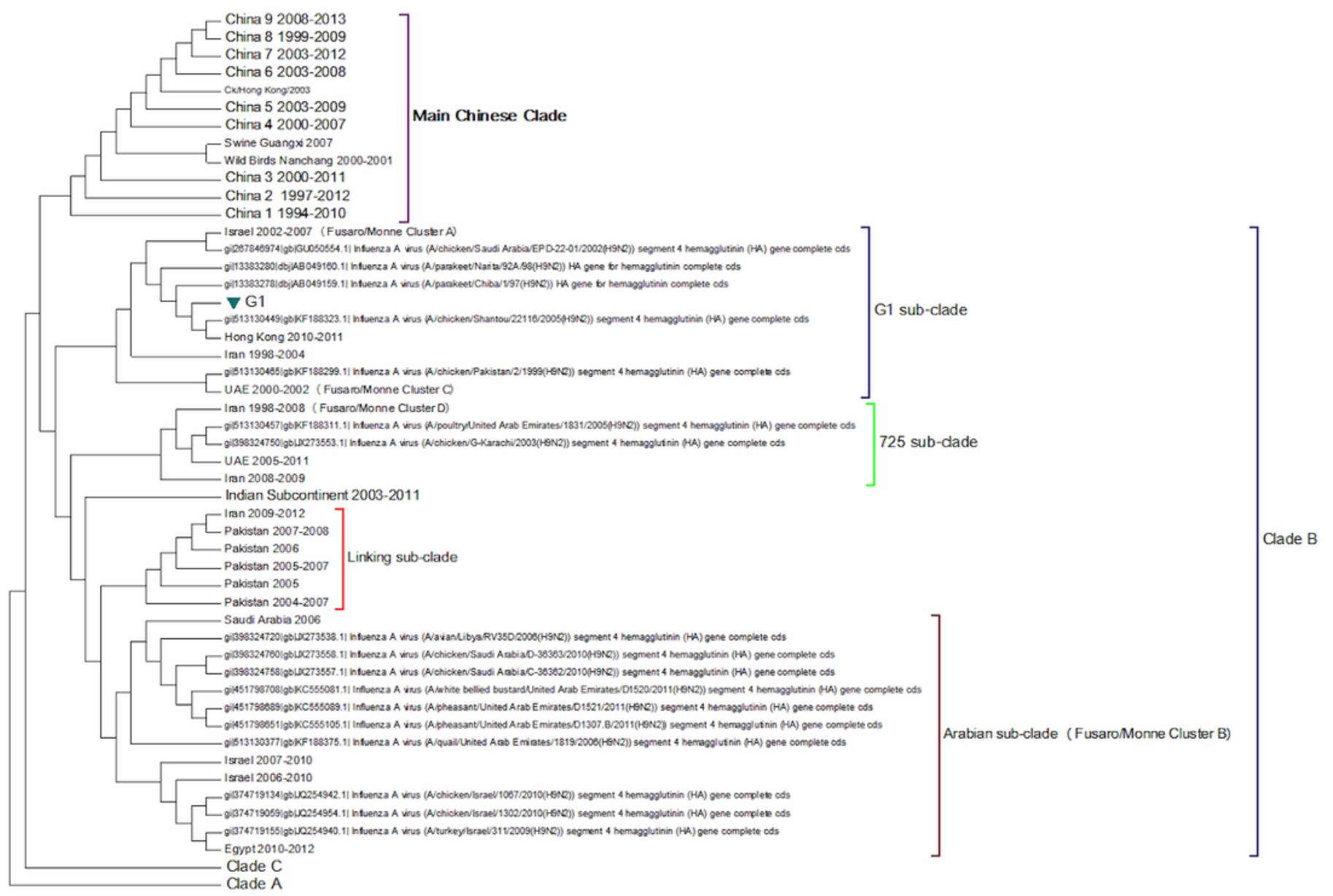




\section{Figure 4}

\section{Clade C}

\section{This is a small clade found between clades A and B}

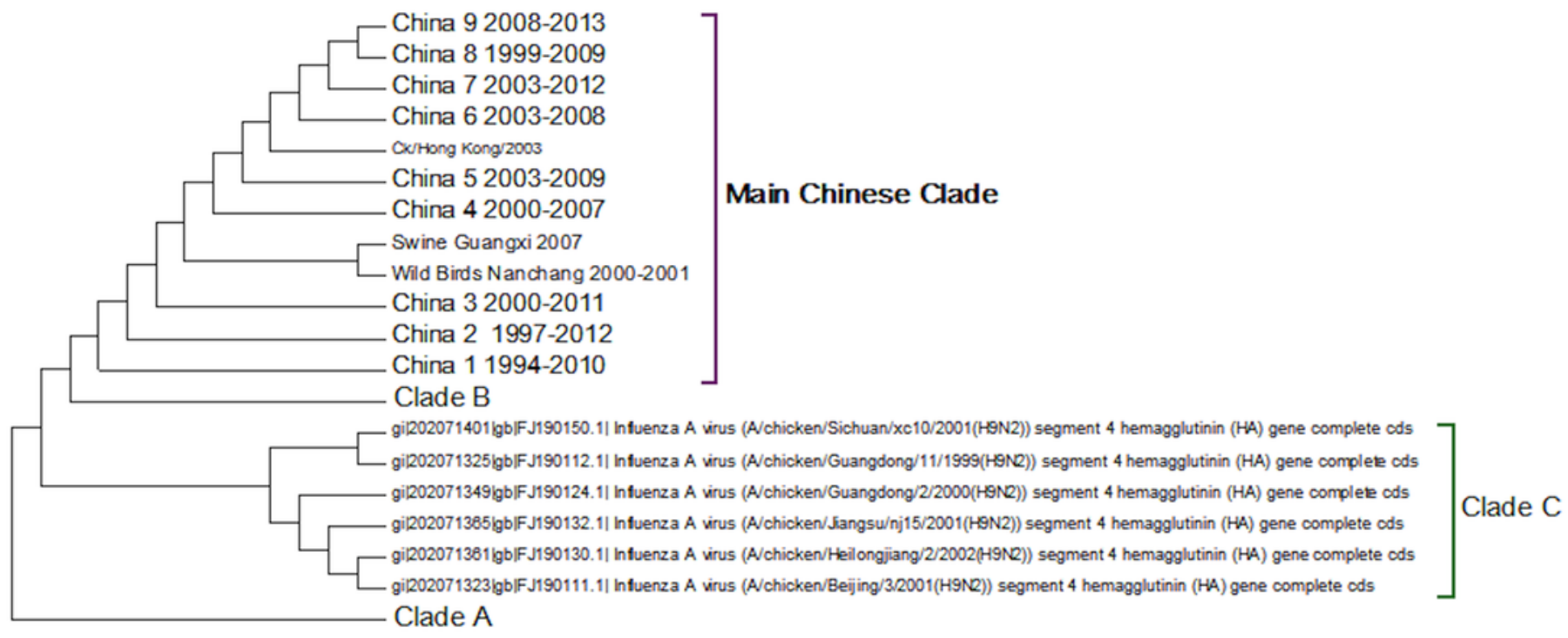


Figure 5

The main Chinese Clade

This contains the Y280 lineage but this has divided into a series of deeply rooted nested clades. 


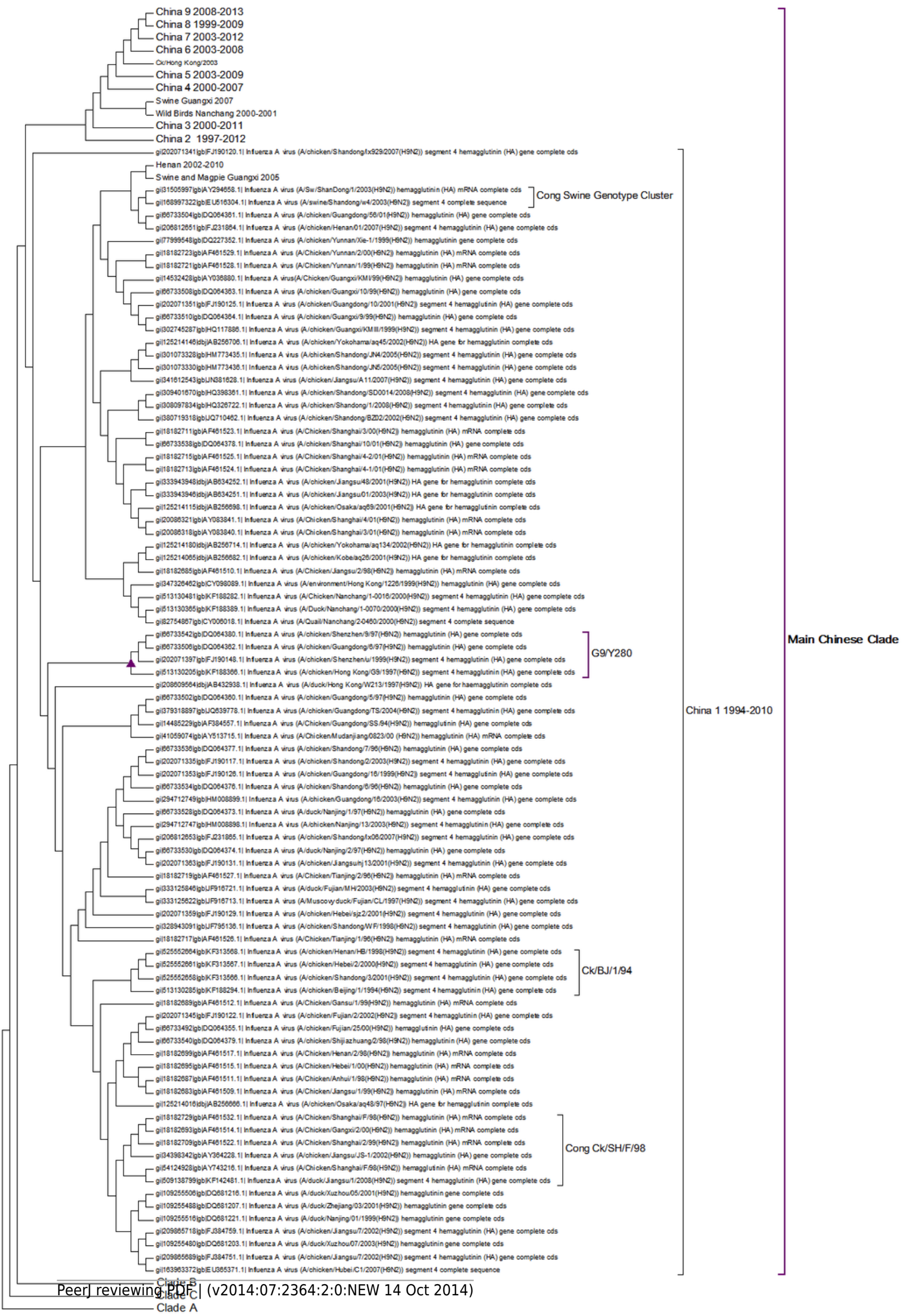


Figure 6

The Korean nucleotide and amino acid phylogenetic trees.

A) The nucleotide phylogenetic tree. B) The amino acid phylogenetic tree.

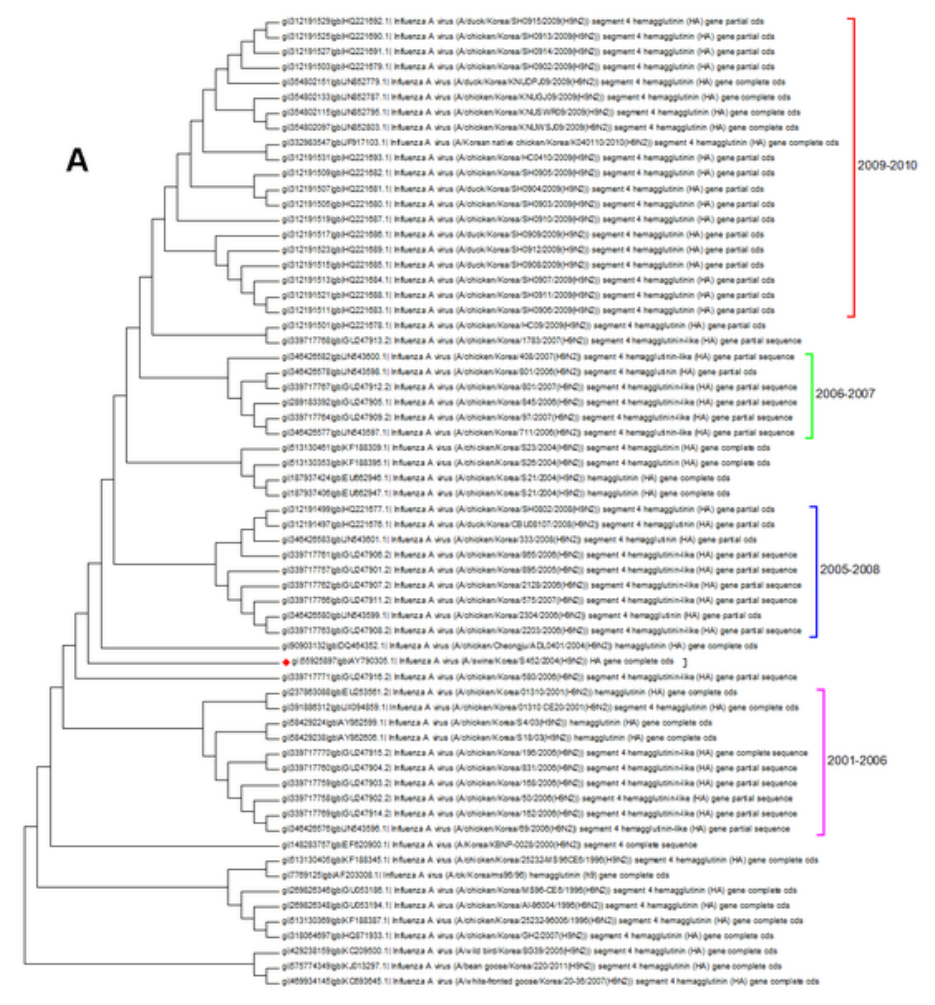

B

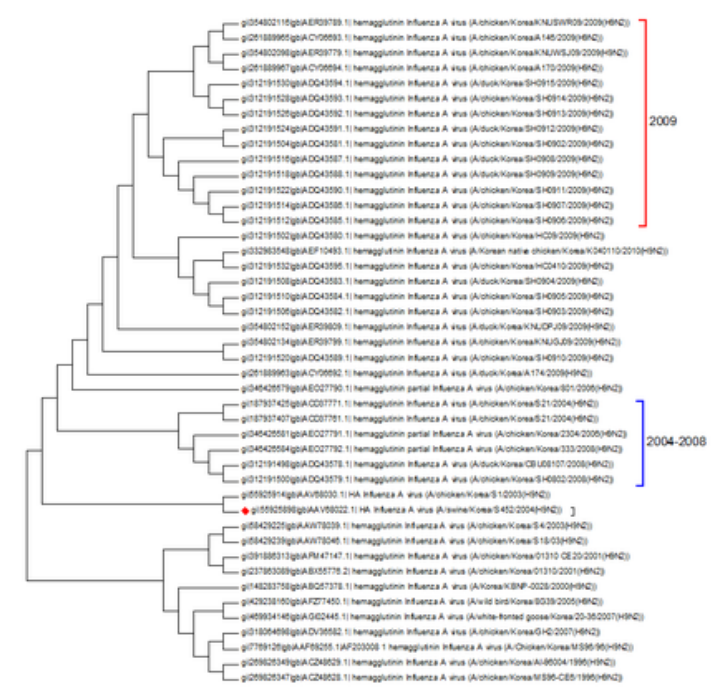


Figure 7

The Iranian and Israeli nucleotide and amino acid phylogenetic trees.

A) The Iranian nucleotide phylogenetic tree, B) The Iranian amino acid phylogenetic tree, C) The Israeli nucleotide phylogenetic tree, D) The Israeli amino acid phylogenetic tree.

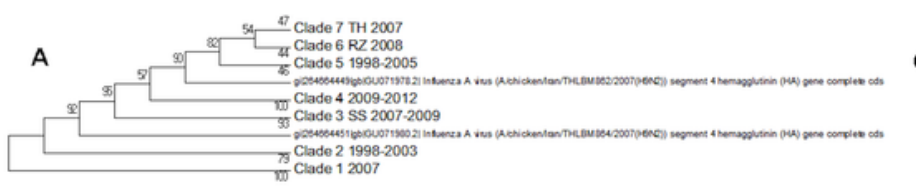
78
Cinde 2 Clade 12007

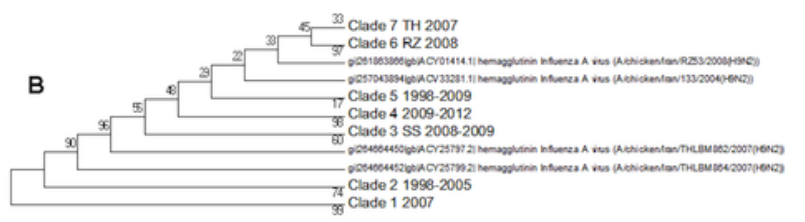

c

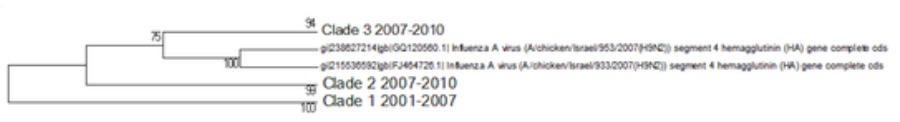

D

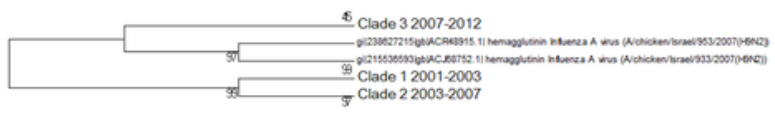


Figure 8

The qual nucleotide and amino acid phylogenetic trees

A) The nucleotide phylogenetic tree, B) The amino acid phylogenetic tree. Numbers on the internal branches are the bootstrap values.
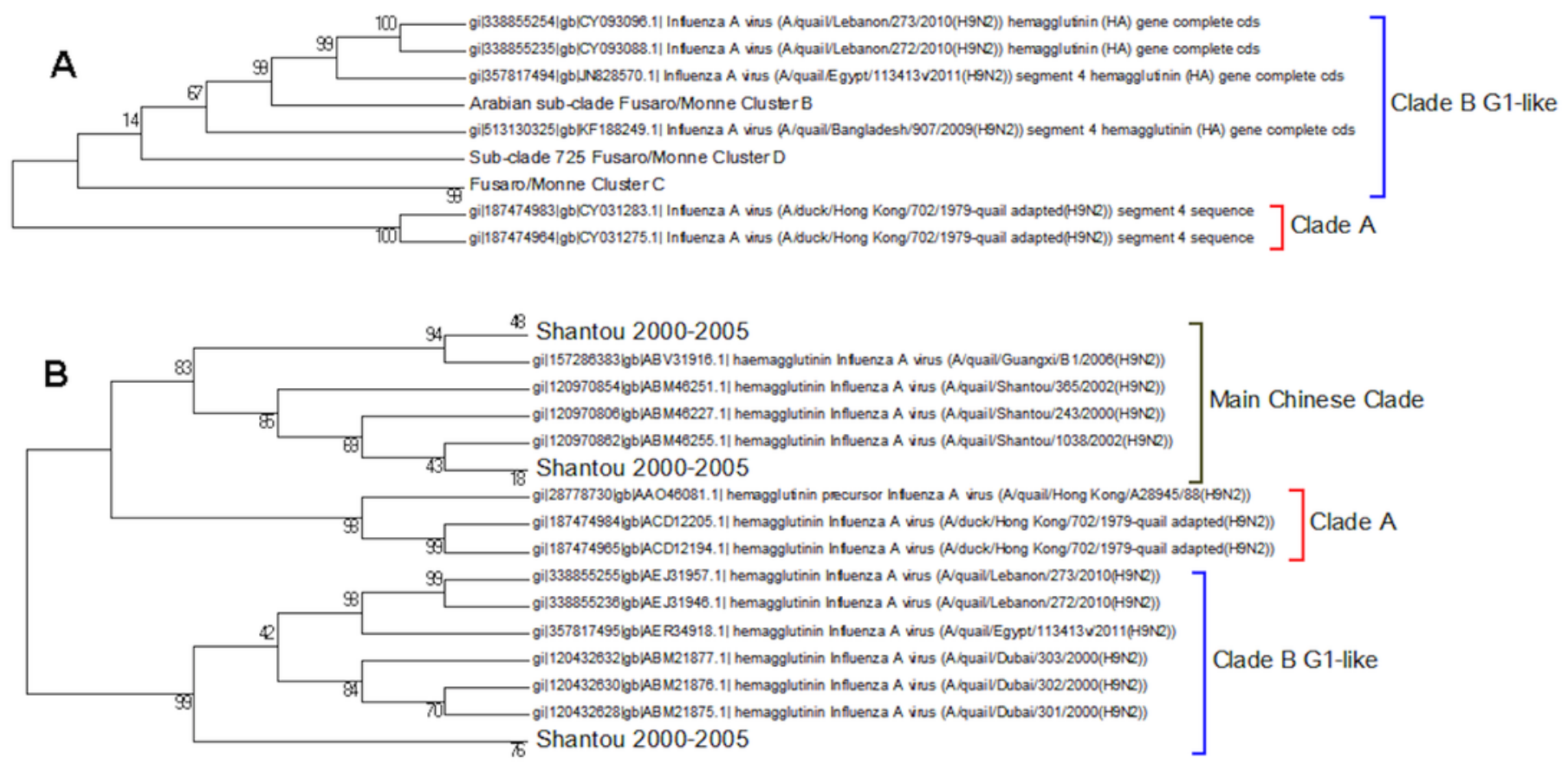


\section{Figure 9}

\section{The duck nucleotide phylogenetic tree}

\section{Numbers on the internal branches are the bootstrap values.}

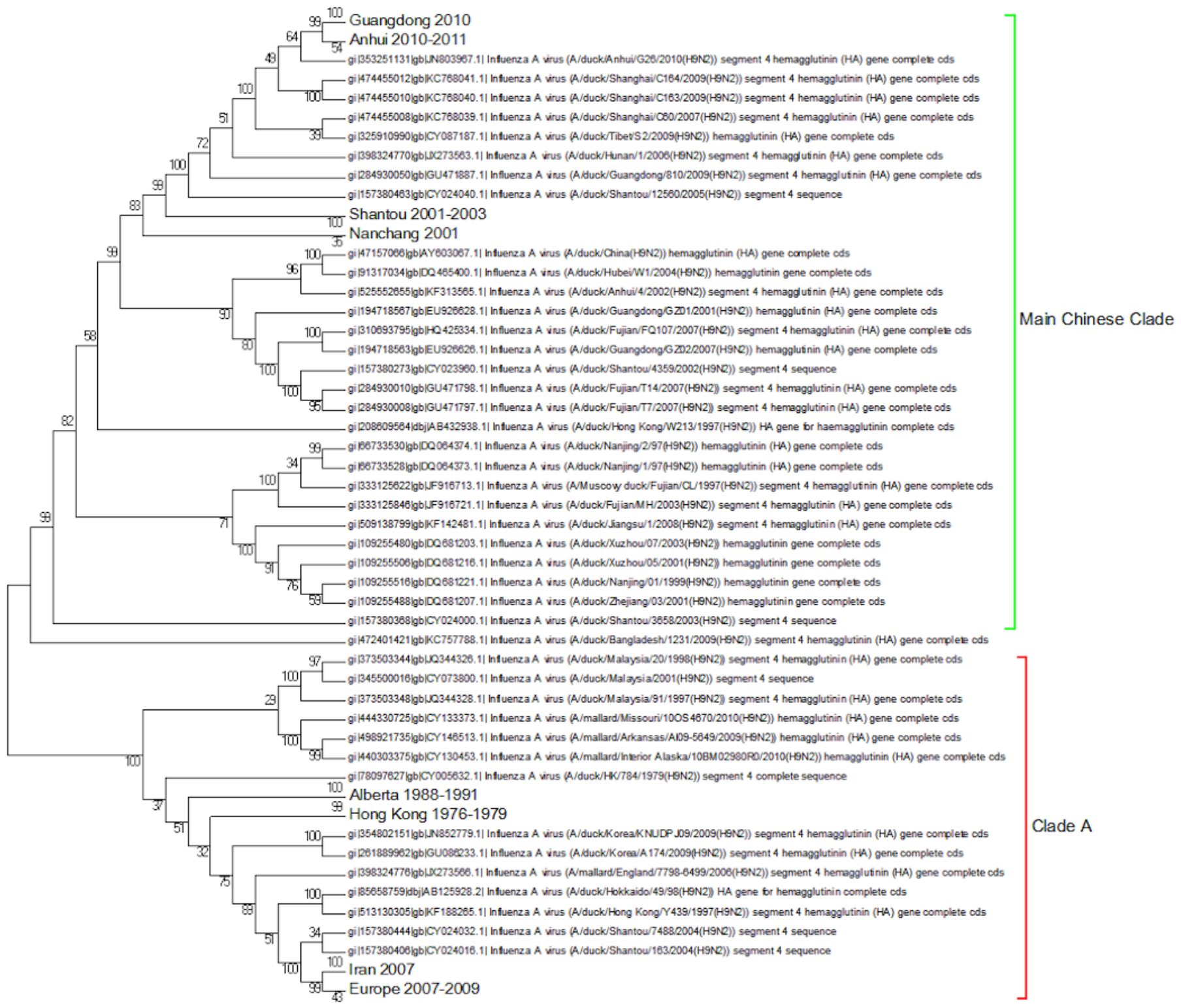




\section{Figure 10}

The swine amino acid phylogenetic tree.

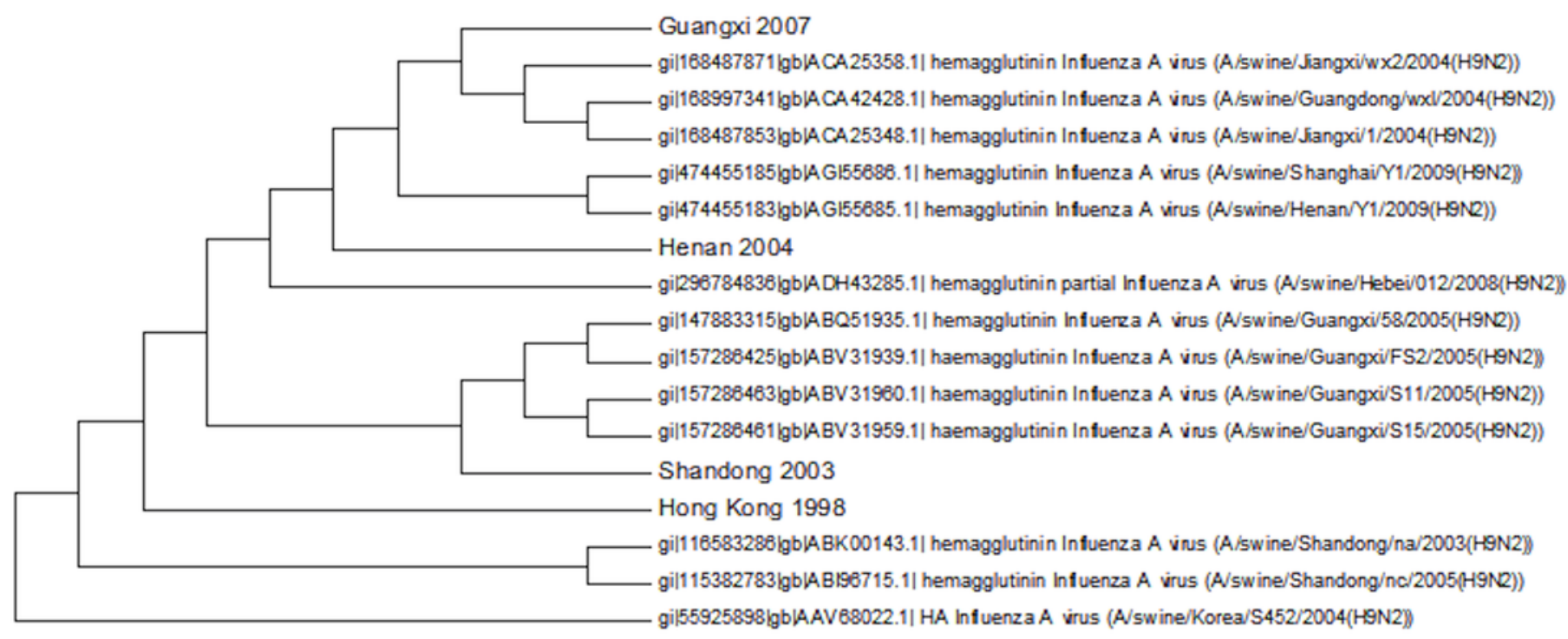




\section{Table 1 (on next page)}

The amino acid differences between clades $A$ and $B$ with respect to the main Chinese clade.

Numbering is for the complete $\mathrm{H} 9$ hemagglutinin protein. Changes are from the conserved sequence (main Chinese clade) to the clade specific sequence. 


\section{PeerJ Reviewing Manuscript}

Numbering is for the complete H9 hemagglutinin protein. Changes are from the conserved sequence (main Chinese clade) to the clade specific sequence.

\begin{tabular}{|c|c|}
\hline Clade A & Clade B \\
\hline $\mathrm{V} 4->\mathrm{T}$ & $\mathrm{V} 3->\mathrm{T}$ \\
\hline $\mathrm{T} 8->\mathrm{A}$ & $\mathrm{V} 15->\mathrm{T}$ or $\mathrm{A}$ \\
\hline $\mathrm{T} 38->\mathrm{I}$ & $\mathrm{N} 40->\mathrm{T}$ \\
\hline A47 -> T & L107 -> T \\
\hline I79 $->V$ & $\mathrm{~S} 121->\mathrm{T}$ \\
\hline R92 -> K & $\mathrm{S} 143->\mathrm{T}$ \\
\hline L122 -> F & $\mathrm{S} 147$-> T \\
\hline $\mathrm{S} 127->\mathrm{N}$ & $\mathrm{S} 158->\mathrm{N}$ \\
\hline Q164 -> H & N167 -> G \\
\hline $\mathrm{R} 180->\mathrm{E}$ & A168 -> L \\
\hline T206 -> A & M187 -> V \\
\hline R335 -> A & N191 -> H \\
\hline S337 -> D & T204 -> I \\
\hline K381 -> E & $\mathrm{R} 205->\mathrm{N}$ \\
\hline V394 -> I & $\mathrm{I} 217->\mathrm{L}$ \\
\hline K473 -> N & D239 -> N \\
\hline \multirow[t]{10}{*}{ N496 -> D } & R294 -> K \\
\hline & T299 -> S \\
\hline & V306 -> I \\
\hline & $\mathrm{N} 313->\mathrm{T}$ \\
\hline & V318 -> I \\
\hline & V333 -> I \\
\hline & S353-> P \\
\hline & I429 -> V \\
\hline & V469 -> M \\
\hline & I537 -> L \\
\hline & \\
\hline
\end{tabular}


PeerJ Reviewing Manuscript 